\title{
Síndrome de Boerhaave
}

\author{
Noemí Pérez Villaverde ${ }^{a}$, Manuel Soto García ${ }^{b}$, Sofía Alemán Villanuevac, \\ Ana Barrios Barberod, Soledad Rodríguez Jiménez ${ }^{\mathrm{a}}$.
}

\begin{abstract}
a Médico de Familia. Centro de Salud "Santa María de Benquerencia” de Toledo. ${ }^{b}$ Médico de Familia. Centro de Salud de Sonseca (Toledo).

'Especialista en

Gastroenterología. Centro Privado. Burgos.

"Médico de Familia. Centro de Salud "Isabel II" de Parla (Madrid)
\end{abstract}

Correspondencia:

Noemí Pérez Villaverde. Centro de Salud "Santa María de Benquerencia" de Toledo.

Correo electrónico:

noemipevi@hotmail.com

Recibido el 28 de septiembre de 2014.

Aceptado para su publicación el 2 de noviembre de 2014.

\section{RESUMEN}

Presentamos el caso de un varón de 37 años que acude a consulta de Atención Primaria por presentar dolor abdominal acompañado de vómitos con sangre y que, tras diferentes valoraciones y una segunda visita al servicio de urgencias, es ingresado en planta, donde se le diagnostica de perforación esofágica espontánea o síndrome de Boerhaave, precisando intervención quirúrgica urgente.

Palabras clave: Perforación esofágica espontánea. Síndrome de Boerhaave. Dolor abdominal.

\section{ABSTRACT}

Boerhaave's Syndrome

We present the case of a 37-year-old man who goes to Primary Care Consultation afflicted with abdominal pain together with blood in vomit. After several evaluations and a second visit to Emergency Service, he is hospitalized, where he is finally diagnosed with Boerhaave Syndrome (spontaneous esophageal perforation), requiring urgent surgery.

Key words: Spontaneous esophageal perforation. Boerhaave syndrome. Abdominal pain.

\section{INTRODUCCIÓN}

El dolor abdominal es un motivo de consulta muy frecuente en Atención Primaria que en no pocas ocasiones precisa valoración urgente en un servicio hospitalario.

La rotura espontánea esofágica (síndrome de Boerhaave) es una entidad poco frecuente. Independientemente del mecanismo de acción, la rotura esofágica se considera como la más grave del tracto digestivo ${ }^{1,2}$, con mortalidad superior al $30 \%{ }^{3}$, situándose en un 20-75 \% la de las roturas espontáneas si el tratamiento no es iniciado a tiempo ${ }^{2}$, llegando a ser fatal en ausencia de tratamiento ${ }^{4}$. Los principales factores pronósticos son el tamaño y la localización de la perforación, la demora en el diagnóstico -con la consecuente contaminación de la pleura y el mediastino- $y$, por supuesto, las condiciones físicas previas del paciente ${ }^{5,6}$.

El retraso en el diagnóstico ocurre hasta en un $50 \%$ de los pacientes ${ }^{7}$, debido a la inespecificidad de los síntomas ${ }^{5}$, no presentándose en muchas ocasiones los síntomas clásicos (vómitos seguidos de dolor torácico o ab- 
dominal alto intenso, en ocasiones irradiado hacia el hombro izquierdo, tras una comida copiosa o ingesta abundante de alcohol) ${ }^{8}$. Además, las alteraciones radiológicas secundarias a la perforación solo están presentes en un $80 \%$ de los casos y suelen aparecer horas o incluso días después del inicio de la clínica, lo cual dificulta aún más el diagnóstico ${ }^{5}$.

Nos decidimos a enviar este caso clínico para hacer hincapié en la necesidad, a pesar de la saturación de nuestras consultas y la de los servicios de urgencias hospitalarias, de estar alerta ante casos cuyo diagnóstico correcto a tiempo es la clave de su pronóstico. El paciente de nuestro caso fue afortunado porque a pesar del retraso en su diagnóstico y tratamiento, su evolución fue favorable.

\section{OBSERVACIONES CLÍNICAS}

Acudió a la consulta un varón de 37 años, sin antecedentes familiares ni personales de interés, refiriendo dolor abdominal intenso en epigastrio asociado a dos vómitos con sangre de escasa cuantía, que se inició mientras estaba comiendo, hacía aproximadamente media hora. En los últimos años había presentado disfagia y molestias abdominales que mejoraban al provocarse el vómito, motivos por los que no había consultado previamente.

En la exploración, presentaba un abdomen blando y depresible, doloroso a la palpación en hipocondrio derecho, pero sobre todo en epigastrio. La auscultación cardiopulmonar fue normal, su presión arterial era de 138/85 mmHg, su frecuencia cardiaca de 84 latidos por minuto, su frecuencia respiratoria de 16 respiraciones por minuto y estaba afebril $\left(36,2^{\circ} \mathrm{C}\right)$.

Ante la sospecha de hemorragia digestiva alta se le derivó al servicio de urgencias hospitalarias. Tras una primera valoración fue dado de alta de este servicio con el diagnóstico de gastroenteritis aguda. Debido a que los síntomas persistían, e incluso se habían agravado a pesar del tratamiento pautado (analgésicos y antieméticos), el paciente acudió de nuevo al día siguiente al servicio de urgencias. En esta ocasión fue ingresado con el diagnóstico de pancreatitis. En planta se objetivó coagulopatía y neumoperitoneo en la radiografía de tórax, por lo que se decidió completar el estudio con un TAC toraco-abdominal objetivándose hallazgos radiológicos compatibles con perforación esofágica a nivel de tercio distal/unión esófago-gástrica, con colección adyacente al esófago, neumoperitoneo y derrame pleural izquierdo secundarios.

Ante estos hallazgos se indicó la intervención quirúrgica (sutura de la perforación con funduplicatura y yeyunostomía) urgente, cursando el postoperatorio favorablemente, por lo que fue dado de alta con posteriores controles en el servicio de cirugía general.

\section{COMENTARIOS}

La rotura espontánea del esófago se define como una dehiscencia de la pared esofágica en zona macroscópicamente sana, habitualmente a consecuencia de un aumento brusco de la presión esofágica, combinado con una presión intratorácica negativa causada por los vómitos o esfuerzos ${ }^{4,9}$.

La historia clínica detallada y la exploración física (sobre todo al inicio de los síntomas) suele ser de poca ayuda en el diagnóstico ${ }^{4}$, como ocurrió con nuestro paciente, que fue derivado a urgencias hospitalarias ante la sospecha de hemorragia digestiva alta y donde fue diagnosticado de gastroenteritis aguda. Los síntomas más habituales son náuseas y vómitos graves, seguidos de dolor agudo retroesternal y abdominal alto, en ocasiones tras un episodio de comida copiosa o bebida abundante de alcohol ${ }^{2,4,7,8}$. Se suceden después rápidamente la odinofagia, taquipnea, disnea, cianosis, fiebre y shock $^{4}$. Por tanto, lo debemos sospechar cuando se asocian síntomas digestivos y respiratorios ${ }^{8,9}$. Es muy característica la triada de Mackler: vómitos, dolor torácico y enfisema subcutáneo, pero sin embargo aparece en muy pocas ocasiones ${ }^{1}$. El enfisema subcutáneo es un hallazgo importante, pero no es muy específico.

El diagnóstico diferencial tenemos que realizarlo con múltiples patologías, entre ellas la pancreatitis (como fue el caso de nuestro paciente), el infarto agudo de miocardio, la disección de aorta, la úlcera perforada, el embolismo pulmonar y la neumonía por aspiración ${ }^{1,7,8}$. 
Las radiografías realizadas en su primera visita a urgencias fueron normales, como sucede en la mayoría de los $\operatorname{casos}^{1,5,9}$, aunque con el paso del tiempo casi siempre presentan alteraciones. Las manifestaciones radiológicas iniciales son neumomediastino o neumoperitoneo, signo que se observó en nuestro paciente durante su ingreso en planta (figura 1). En las roturas cervicales se observa aire en los tejidos blandos del espacio prevertebral. Horas o días después puede aparecer derrame pleural con o sin neumotórax, ensanchamiento mediastínico y enfisema subcutáneo ${ }^{4}$.

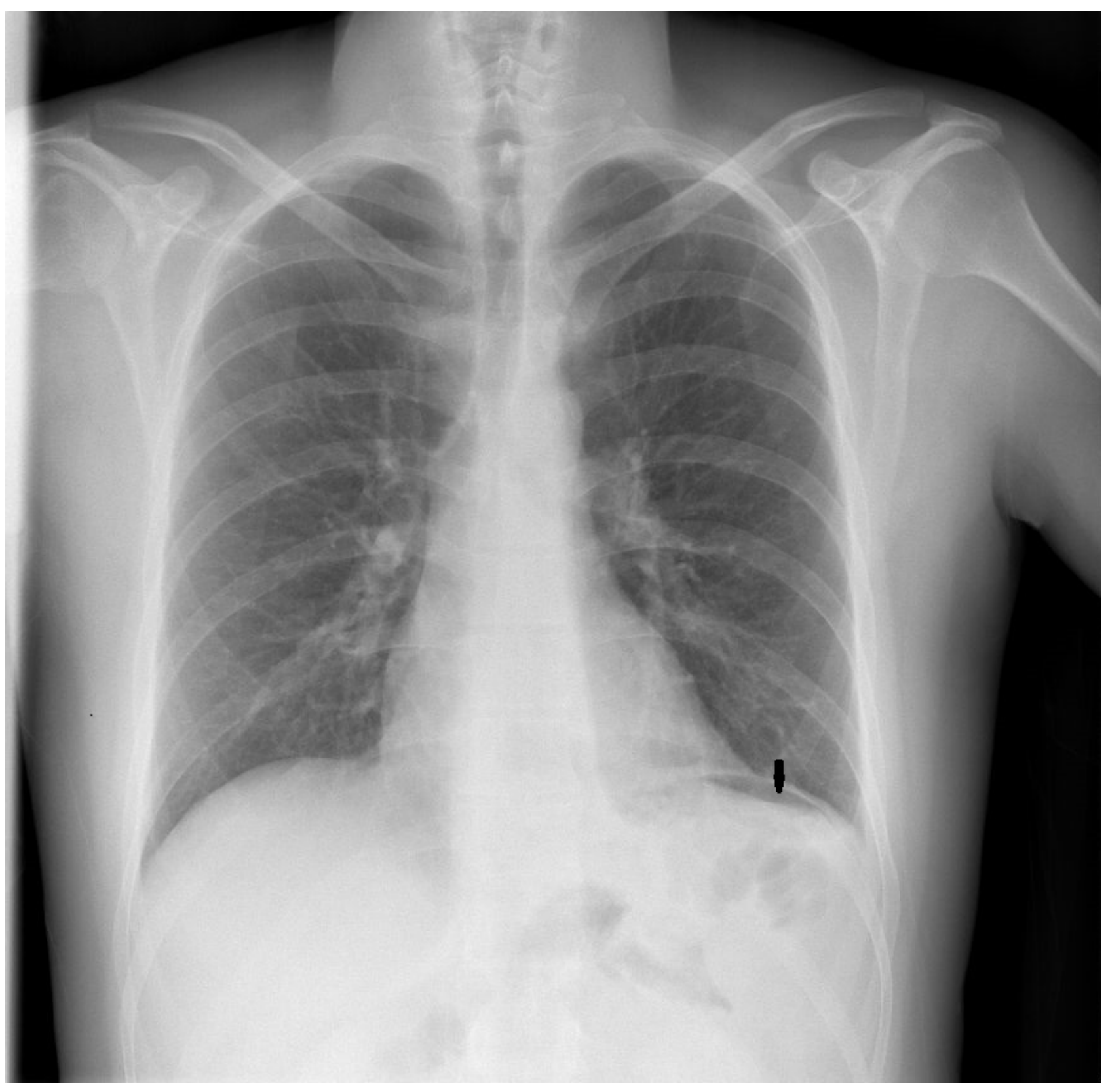

Figura 1. Neumoperitoneo 
En los pacientes con clínica y radiografía de tórax sospechosas debemos solicitar rápidamente un TAC y/o un esofagograma. En el TAC pueden visualizarse colecciones líquidas $O$ niveles hidroaéreos periesofágicos sugestivos de perforación, pero no se puede precisar la localización y tamaño de la perforación ${ }^{2}$, lo que se hace con el esofagograma, usando un contraste hidrosoluble (gastrografin) 4 . La utilización de la esofagoscopia sigue siendo polémica, ya que la insuflación de aire puede aumentar el tamaño de la lesión y pasar aire a mediastino ${ }^{4}$. En nuestro caso fue suficiente la realización del TAC para el diagnóstico, puesto que con los hallazgos encontrados se creyó necesaria la intervención urgente con el fin de reparar la lesión.

Hay poca evidencia para recomendar un tratamiento, que puede ser conservador o quirúrgico, así como una técnica quirúrgica u otra ${ }^{2}$. Actualmente las técnicas quirúrgicas y los cuidados intensivos han reducido la mortalidad incluso cuando se retrasa la cirugía, sin embargo, cuando esta se produce más allá de las 24 horas sigue asociándose a un incremento de mortalidad ${ }^{4}$.

Ante este caso debemos ser conscientes de que existen patologías en las que la historia clínica detallada e incluso las pruebas complementarias al inicio puede que no nos orienten hacia un diagnóstico determinado, pero aun así debemos ser muy cuidadosos en su realización e interpretación, evitando errores importantes como en el caso de nuestro paciente, al que se le diagnosticó en un principio de una patología banal, puesto que el retraso en su diagnóstico puede ser vital para el paciente.

\section{BIBLIOGRAFÍA}

1. Hiroyuki Ando, Yoshinori Shitara, Keigo Hara, Yasushi Mogami, Tsutomo Kobayashi, Toshiki Yajima et al. Successful surgical treatment o spontaneous of the esophagus diagnosed two dayes after onset. Case Rep Gastroenterol. 2012;6:260-5.

2. Tonolini M, Bianco R. Spontanuous esophageal perforation (Boerhaave syndrome): Diagnosis with CT-esophagography. J Emerg Trauma Shock. 2013;6:58-60.

3. Ríos Zambulido A, Martínez de Haro LF, Ortiz Escandell MA, Durán H, Munitiz Ruiz V, Parrilla Paricio. Perforaciones esofágicas. Presentación de 23 casos. Gastroenterol Hepatol. 2000;23:379-83.

4. Triadafilopoulos G. Boerhaave's syndrome: Effort ruptura of the esophagus. [Monografía en internet]: UptoDate; Grove S, 2013 [acceso 25 de julio de 2013]. Disponible em: http/www.uptodate.com.

5. Gutiérrez Macías A, Zubeldia Sánchez E, Barbero Blanco E, García Cuevas M, Soto Beobide A, Agirreazabal Rementería $\mathrm{J}$ et al. Perforación esofágica espontánea o síndrome de Boerhaave. Una causa de dolor torácico potencialmente mortal. Emergencias. 1998;10:196-9.

6. Van Gijn J, Gijselhart JP. Boerhaave and his syndrome. Ned Tijdschr Geneeskd. 2013;157(4):A5460.

7. Tamatey MN, Sereboe LA, Tettey MM, Entusa-Mensah K, Gyan B. Ghana Med J. 2013;47:53-5.

8. Teh E, Edwards J, Duffy J, Beggs D. Boerhaaver's syndrome: a review of management and outcome. Interactive cardiovascular and thoracic surgery. 2007; 6:640-3.

9. Sánchez Fernández J, Jiménez López M, Lozano Sánchez F, Varela Simó G, Cuadrado Idoyaga F, Gómez Alonso A. Rotura espontánea de esófago (síndrome de Boerhaave). Un caso de presentación atípica. Cir Esp. 2000;67:308-9. 